\begin{tabular}{|c|c|c|c|}
\hline Class & Compound & VIP Score & $\begin{array}{l}\text { Fold-change } \\
\text { Modern vs } \\
\text { ancient }\end{array}$ \\
\hline \multirow[t]{4}{*}{ Alkaloids and derivatives } & (R)-N-methylcoclaurine & 1.75084 & 3.92 \\
\hline & cathinone & 1.72991 & 1.68 \\
\hline & gramine & 1.66464 & -6.17 \\
\hline & methylnicotinate & 1.60727 & 0.98 \\
\hline \multirow{5}{*}{ Hormones } & 16,17-dihydro-16 $\alpha, 17$-dihydroxy gibberellin $\mathrm{A}_{12}$ & 1.69372 & 2.98 \\
\hline & 3-hydroxy-2-oxo-(indol-3-yl)acetate & 1.61908 & 0.05 \\
\hline & 7-hydroxy-2-oxindole-3-acetate & 1.6084 & 1.32 \\
\hline & benzyladenine-N-glucoside & 1.75241 & 5.22 \\
\hline & dihydrozeatin-9-N-glucoside-O-glucoside & 1.70651 & 1.31 \\
\hline \multirow{8}{*}{ Phenolic compounds } & (S)-colchicine & 1.68329 & 1.50 \\
\hline & 5-O-caffeoylshikimate & 1.68429 & 0.55 \\
\hline & 6-C-glucosyl chrysin & 1.68081 & 1.15 \\
\hline & $\begin{array}{l}\text { cyanidin 3-O-(6-O- } \beta \text {-D-glucosyl-2-O- } \beta \text {-D-xylosyl- } \beta \text {-D-galactoside)// cyanidin 5-O- } \beta \text {-D- } \\
\text { glucoside 3-O- } \beta \text {-D-sambubioside }\end{array}$ & 1.84388 & 18.44 \\
\hline & cyanidin 3-O- $\beta$-D-caffeoylglucoside & 1.75829 & 0.79 \\
\hline & cyanidin 3-O- $\beta$-D-sambubioside & 1.75747 & 0.59 \\
\hline & dalpatein 7-O- $\beta$-D-apiofuranosyl-(1->6)- $\beta$-D-glucopyranoside & 1.73921 & 0.39 \\
\hline & $\begin{array}{l}\text { delphinidin 3,5-di-O- } \beta \text {-D-glucoside// delphinidin 3-O-sophoroside// quercetin 3,4'-O- } \\
\text { diglucoside// delphinidin 3,7-di-O- } \beta \text {-D-glucoside// quercetin 3-O-sophoroside// quercetin- } \\
\text { 3-gentiobioside// quercetin A-O-diglucoside// quercetin 3-O- } \beta \text {-D-glucosylgalactoside }\end{array}$ & 1.70699 & 1.30 \\
\hline
\end{tabular}


isorhamnetin 3-O-(6"-O-feruloyl)-glucoside $\quad 1.86575$

isorhamnetin 3-O-(N-O-p-coumaroyl)-glucoside// kaempferol 3-O-(6l-O-feruloyl)-

1.67972

glucoside"

1.75495

coumaroylglucoside

1.74837

0.45

isovitexin 2"-O- $\beta$-D-glucoside

1.83728

2.16

isovitexin-7-O-glucosyl-2"O-rhamnoside

1.61968

glucoside// isovitexin 7-O-glucoside// pelargonidin 3-O-sophoroside// genistin 7-

gentiobioside// apigenin-7-O-gentiobioside// pelargonidin 3,7-di-O- $\beta$-D-glucoside//

isovitexin 7-O-galactoside// luteolin 7-O-neohesperidoside// kaempferol 3-O-rhamnoside-

7-O-glucoside// 4-(2-aminophenyl)-2,4-dioxobutanoate// cyanidin-3-O-rutinoside

kaempferol-3-glucoside-7-rhamnoside

1.75105

0.48

maysin

1.74213

0.61

pelargonidin-3-O-rutinoside-5-O- $\beta$-D-glucoside

1.7845

1.62

peonidin 3,5-diglucoside

1.67952

2.33

quercetin 3-O-(4"-O-p-coumaroyl)-glucoside

1.76631

2.51

quercetin 3-O-(N-O-p-coumaroyl)-glucoside

1.77225

2.28

quercetin-3-rhamnoside-7-rhamnoside// vicenin-2// vitexin 2"-O- $\beta$-D-glucoside//

1.86977

24.63

\section{Serotonin amide} rhamnosylisoorientin

\section{Terpenoids}

cinnamoylserotonin

1.75397

1.79729

3.14

$\mathrm{N}$-acetyl-serotonin

1.61702

19.58

$9 \alpha$-copalyl diphosphate

1.75957

0.65

iridotrial

1.72091

0.58

steviolbioside// rubusoside

1.25

(+)-copalyl diphosphate// 2-cis,6-trans,10-trans-geranylgeranyl diphosphate//

Others geranylgeranyl diphosphate// (13E)-labda-7,13-dien-15-yl diphosphate// (-)-ent-copalyl

1.62715

0.25

diphosphate

(R)-prunasin

1.79616

0.67

1-[18-hydroxyoleyl]-2-lyso-phosphatidate

1.79751

1.76

1-O-4-hydroxybenzoyl- $\beta$-D-glucose

1.7962

0.71 
3,5-di-C-glucosyl-2,4,4',6-tetrahydroxydibenzoylmethane

\begin{tabular}{ll}
1.78858 & -3.46 \\
1.61786 & -11.33 \\
1.61747 & -9.45 \\
1.87754 & 20.28 \\
1.76168 & 1.79 \\
1.69694 & 1.51 \\
1.6134 & 1.29 \\
1.82529 & 2.57 \\
1.66065 & 1.55 \\
1.86579 & 16.87 \\
1.61097 & -0.12 \\
1.85959 & 1.68 \\
1.73224 & 0.35 \\
1.76599 & 2.28 \\
1.61281 & 0.92 \\
1.68643 & 0.53 \\
1.84421 & 17.19 \\
1.7739 & 0.63 \\
1.66501 & -1.18 \\
1.61281 & 0.92 \\
1.70485 & 0.51 \\
1.7271 & 17.56 \\
\hline &
\end{tabular}

3-cyano-L-alanine

3-ureidopropanoate

4-(1-methyl-2-pyrrolidinyl)-3-oxobutanoate methyl ester

7-hydroxy-2-oxindole-3-acetate glucoside

7-methylinosine

adipate

allocryptopine

benzoyl- $\beta$-D-glucopyranose

bisorganyltrisulfane

chorismate/isochorismate

diethylpyrocarbonate

ferric biliverdine-IX- $\alpha$

glutathione disulfide

homoglutathione

indole-3-carboxylate

L-galactono-1,4-lactone// 3-keto- $\beta$-D-galactose// L-sorbosone// D-glucono-1,5-lactone// Lgulono-1,4-lactone// L-sorbosone-N-lactone

phenylethyl acetate// eugenol// 4-hydroxyphenylbutan-2-one//

S-(2-aminoethyl)-L-cysteine

S-methylglutathione

xylogalacturonan

1.7271

17.56 